\title{
Orientation illusions vary in size and direction as a function of task-dependent attention
}

\author{
Roberta DAINI \\ Università degli Studi di Milano-Bicocca, Milan, Italy \\ AND \\ Peter Wenderoth \\ Macquarie University, Sydney, New South Wales, Australia
}

\begin{abstract}
Adding an upright inner square frame to an outer tilted square frame causes a central rod's perceived orientation to be directionally opposite the usual rod-and-frame illusion (RFI). Zoccolotti, Antonucci, Daini, Martelli, and Spinelli (1997) attributed this double RFI (DRFI) to Rock's (1990) hierarchical organization principle. In Experiment 1, this explanation predicted results for small $\left(11^{\circ}\right)$ but not larger $\left(22^{\circ}\right.$ and $\left.33^{\circ}\right)$ outer frame orientations. In two experiments with the DRFI, bottom-up, goal-driven attention was varied and direct and indirect measures of the framework's influence were compared. In Experiment 2, the RFI angular function was compared with two other DRFI conditions: a direct measure of perceived rod orientation and an indirect measure of the inner frame. These conditions induced directionally opposite effects. In Experiment 3, direct and indirect measures of the inner frame's perceived tilt were compared. Angular functions differing in size and direction were obtained. Experiment 4 replicated the previous results, using a different psychophysical procedure. All the results were consistent with the hierarchical organization mechanism but suggested different processing strategies due to different attentional weights. They were also consistent with other recent findings based on the Bayesian approach to accounts of illusory phenomena (e.g., Jazayeri \& Movshon, 2006, 2007; Weiss, Simoncelli, \& Adelson, 2002).
\end{abstract}

The rod-and-frame illusion (RFI) refers to the misjudgment of the orientation of a gravitationally vertical $\left(0^{\circ}\right)$ rod when it is surrounded by a tilted square frame (Witkin $\&$ Asch, 1948), as shown in Figure 1A. In central vision, the amplitude and direction of vertical setting errors vary as a function of frame orientation and have been attributed to a global orientation mechanism based on the influence of the four axes of bilateral symmetry of the square frame, with two of the axes defined by the real main lines of the frame and two defined by the virtual diagonal axes (e.g., Beh, Wenderoth, \& Purcell, 1971; Wenderoth \& Beh, 1977; Wenderoth, van der Zwan, \& Johnstone, 1989; Wenderoth, van der Zwan, \& Williams, 1993). Influenced by the inducer (frame) symmetry axis closest to vertical, subjects tend to perceive the rod as vertical when it is tilted in the same direction as the inducing frame at small frame orientations between $0^{\circ}$ and about $22.5^{\circ}$, at which a real main-line frame axis is closest to vertical (repulsion effect), and when it is tilted in the opposite direction (attraction effect) at larger frame orientations between $22.5^{\circ}$ and $45^{\circ}$, at which a virtual diagonal axis is closest to vertical. Referring to clockwise (CW) tilts as +ve and counterclockwise (CCW) tilts as -ve, the typical angular function of the RFI (Figure 1C) shows a peak repulsion $(+v e)$ effect at around $+11^{\circ}$ of frame orientation and a peak attraction $(-v e)$ effect at about $+33^{\circ}$, with the zero crossing around $+22^{\circ}$ (e.g., Beh et al., 1971; Wenderoth, 1974; Wenderoth \& Johnstone, 1987).

The double RFI (DRFI) refers to the misjudgment of an upright rod when it is surrounded by two concentric square frames, as in Figure 1B (Daini, Wenderoth, \& Smith, 2003; Spinelli, Antonucci, Daini, Martelli, \& Zoccolotti, 1999; Zoccolotti, Antonucci, Daini, Martelli, \& Spinelli, 1997). When the outer square frame is tilted $+11^{\circ}$ and the inner square frame is upright $\left(0^{\circ}\right)$, a paradoxical rod misjudgment occurs, so that an apparent attraction, rather than a repulsion, effect is obtained. Rock's (1974) concept of frame of reference predicts that only the more external frame will affect the perceived rod tilt, so that a repulsion effect would be predicted to occur. Zoccolotti et al. interpreted this unexpected apparent DRFI attraction effect in terms of another of Rock's (1990) concepts - namely, the hierarchical organization process, in which an object is perceived in terms of its relation only to its immediately adjacent frame of reference. That is, the perceptual system should tend to organize units of the field hierarchically, from the outer to the inner stimulus. Each element in the field should be "perceived primarily in terms of its changing relation to its immediate frame of reference rather than to any external frame" (Rock, 1990, p. 255).

R. Daini, roberta.daini@unimib.it 
A

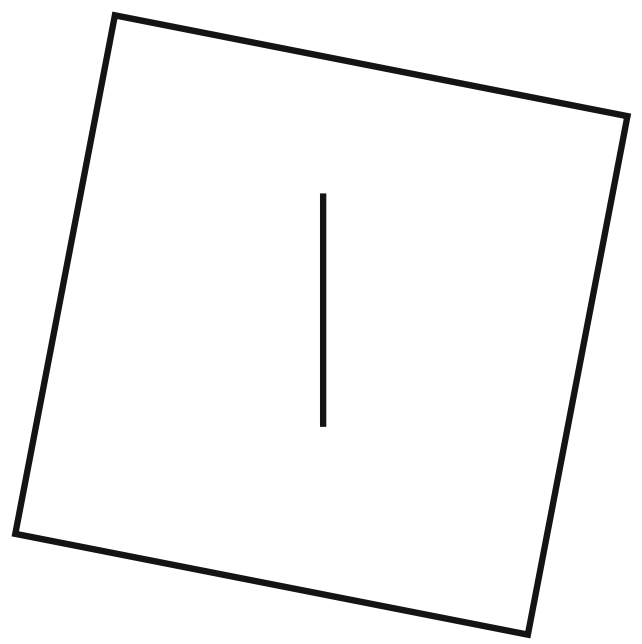

B

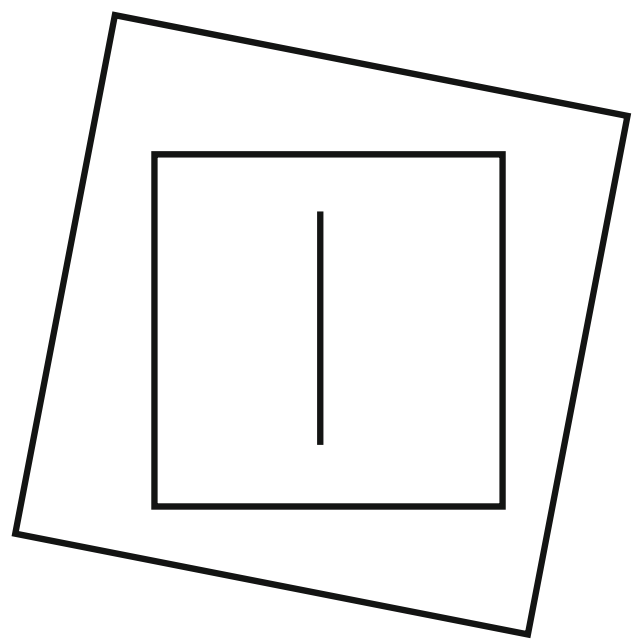

C

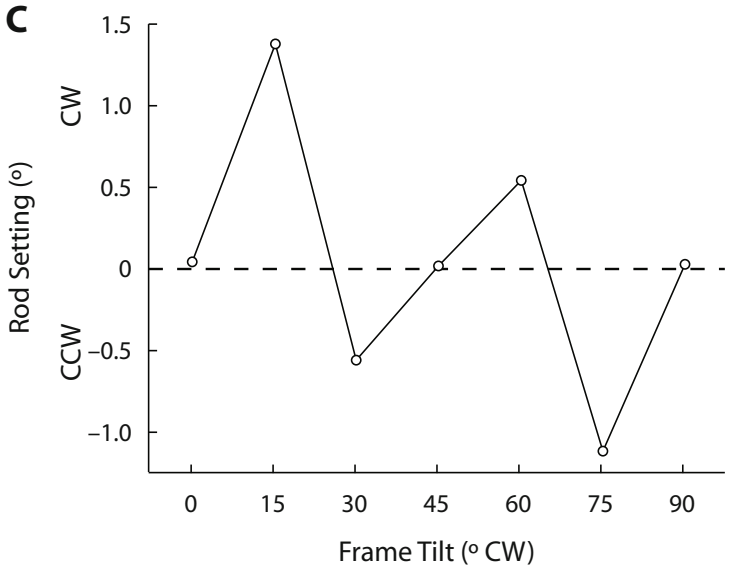

Figure 1. (A) The standard rod-and-frame illusion (RFI), with the frame tilted $11^{\circ}$ clockwise $(\mathrm{CW})$. (B) The double RFI with the outer frame tilted $11^{\circ} \mathrm{CW}$ and the inner frame vertical $\left(0^{\circ}\right)$. (C) The angular function of the RFI (Beh, Wenderoth, \& Purcell, 1971). CCW, counterclockwise.
According to this hierarchical organization interpretation, the outer frame in the DRFI induces a misperception of the inner frame, which, and only which, in turn affects the rod. Specifically, the $+11^{\circ}$-tilted outer frame makes the vertical inner frame appear tilted $\mathrm{CCW}$, and the latter makes the rod appear tilted CW. Thus, what appears to be an attraction effect from the outer CW tilted frame is actually a repulsion from the perceived $\mathrm{CCW}$ tilt of the vertical inner frame.

Spinelli et al. (1999) measured the size of the illusion obtained both when the test stimulus was a square frame and when it was a rod and found identical illusions in the two cases, $\left(+1.4^{\circ}\right.$ and $+1.3^{\circ}$, respectively $)$ consistent with the hierarchical organization prediction that a $\mathrm{CW}$-tilted outer frame makes a vertical inner frame appear tilted $\mathrm{CCW}$. They also showed that a small actual inner frame tilt of $1^{\circ}-2^{\circ} \mathrm{CCW}$ from vertical induced significant repulsion illusions for a test rod. Their quantitative analysis was consistent with the interpretation of a concentric effect from the outermost to the innermost frame and from the inner frame to the rod, as predicted by the hierarchical organization hypothesis. However, all previous studies of the DRFI have used only one orientation of the outer inducing stimulus - namely, $+11^{\circ}$ (Daini et al., 2003; Spinelli et al., 1999; Zoccolotti et al., 1997).

What might be predicted for the DRFI when the outer square frame is tilted more than $11^{\circ}$ ? The prediction would be that at $22^{\circ}$, for which zero effect occurs in the standard RFI, no errors should be obtained, because the outer frame will have no effect on the vertical inner frame. However, "anomalous" repulsion effects will be expected at $+33^{\circ}$ outer frame tilt, for which the single-frame illusion usually induces attraction effects, because the CW-tilted outer frame will induce an attraction effect on the inner frame, a CW-perceived tilt, causing the rod to appear to be tilted $\mathrm{CCW}$, as if repelled from the outer frame. Another prediction would be that the amount of illusion in the doubleframe condition will always be smaller than that in the usual RFI conditions, because the tilt induced on the inner frame is very small.

The first experiment was run to explore the angular function of the DRFI illusion, changing only the outermost frame orientation. The results of this first experiment did not confirm the prediction made on the basis of the hierarchical organization hypothesis and encouraged us to perform two other experiments and to propose a new interpretation of the DRFI effects.

The second and third experiments were designed to discover the effect of the outer frame on the inner frame. A fourth experiment was conducted, using a different method, to replicate the findings and to generalize our results.

In some DRFI experiments, for each of three outer frame tilts $\left(11^{\circ}, 22^{\circ}\right.$, and $\left.33^{\circ}\right)$, we varied the tilt of the rod and measured the point of subjective verticality (PSV) of the rod. We refer to this paradigm as DRFI (RR), where the first letter in the parentheses refers to the variable stimulus component and the second refers to the judged component. Similarly, in the DRFI (FR) paradigm, for each of the three outer frame tilts $\left(11^{\circ}, 22^{\circ}\right.$, and $\left.33^{\circ}\right)$ the inner frame 
tilt varied, but we asked the observers to judge the rod. In this case, because the rod was actually truly vertical always but the various tilts of the inner frame made it appear tilted, this was an indirect measure of the PSV of the inner frame. Finally, in the paradigm DRFI (FF) for each of the three outer frame tilts $\left(11^{\circ}, 22^{\circ}\right.$, and $\left.33^{\circ}\right)$, the inner frame tilt varied, and we directly measured the PSV of the inner frame.

\section{EXPERIMENT 1}

In Experiment 1, we compared the angular function of the standard RFI with that of the DRFI (RR). Subjects were asked to judge the PSV of a rod when it was surrounded by a single tilted square frame (standard RFI) and when it was surrounded by an inner upright square frame and an outer tilted square frame (DRFI [RR]).

According to previous studies and the hierarchical organization concept, predictions were that the standard display would induce a repulsion effect for small frame orientations $\left(11^{\circ}\right)$ and an attraction effect for large orientations $\left(33^{\circ}\right)$, whereas opposite and smaller illusions would be produced by the double-frame display.

\section{Method}

Subjects. Twenty-one 20- to 30-year-old students (11 of them male and 10 female) from the University of Rome "La Sapienza" participated in the experiment as volunteers. All had normal or corrected-to-normal vision.

Stimuli, Apparatus, and Procedure. The stimuli were drawn using Adobe Photoshop and were presented using SuperLab software on a 21-in. Sony Trinitron display monitor interfaced with a Macintosh Power 8200 computer. All the stimuli were black on a white background. A rod subtending $2^{\circ}$ of visual angle was surrounded by one square (standard RFI; see Figure 1A) or by two concentric squares (DRFI [RR], see Figure 1B). In both conditions, the side of the inducing (outermost) square subtended $12^{\circ}$ of visual angle and could be tilted $11^{\circ}, 22^{\circ}$, or $33^{\circ}$, either CCW or CW. In the DRFI (RR) condition, an upright inner square, with sides of $6^{\circ}$, was added. The variable stimulus (the rod) was presented at 13 different orientations (from $-6^{\circ} \mathrm{CCW}$ to $+6^{\circ} \mathrm{CW}$, with $1^{\circ}$ step intervals). The combination of two experimental conditions (RFI and DRFI [RR]), 13 rod tilts, and six outer frame orientations produced a total of 156 trials. The stimuli were presented to all the subjects in the same quasirandom sequence. The sequence of presentation was not blocked by condition. The subjects' responses were recorded using two buttons on a keyboard to indicate whether the rod appeared to be tilted to the left or the right of vertical, using a twoalternative forced choice (2AFC) method. The subjects were seated in a darkened laboratory in which all external cues to vertical were removed by attaching a black cardboard $20^{\circ}$-diameter mask to the display monitor. The stimuli were presented at a distance of around $57 \mathrm{~cm}$, so that $1 \mathrm{~cm}$ on the screen subtended $1^{\circ}$ of visual angle.

\section{Results}

The means and standard errors of obtained illusions are shown in Figure 2.

The data were analyzed using the unidimensional Guttman scale. This allowed establishing the PSV as the point of inversion between CCW and CW responses. The reliability of the measures was assessed by the coefficient of reproducibility (Dunn-Rankin, 1983).

In the single- and double-frame conditions, all of the coefficients of reproducibility were above 0.90 , indicating that the observed values were a good approximation of a perfect scale.

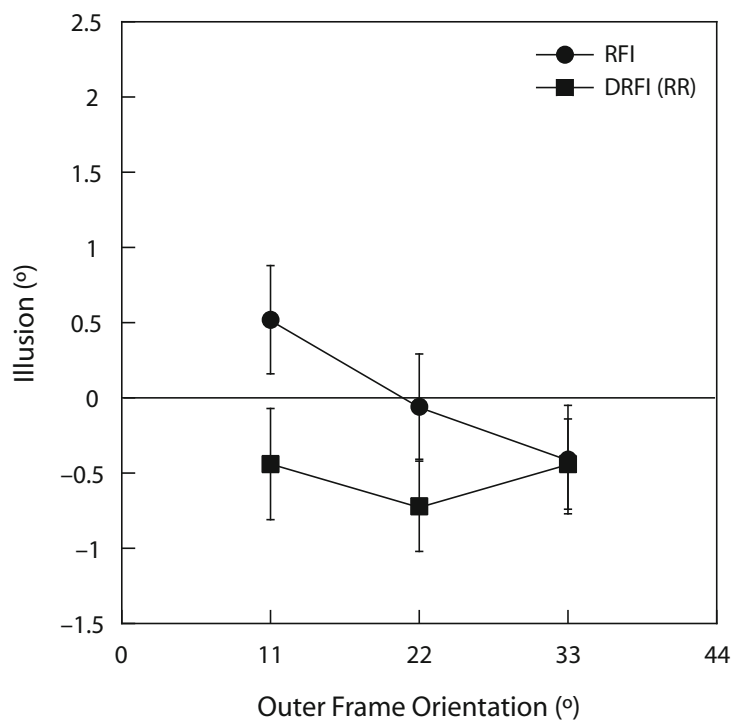

Figure 2. Means and standard errors of the illusions obtained in Experiment 1, as a function of outer frame orientation. A positive sign for the illusion stands for the repulsion effect, and a negative sign indicates an attraction effect.

PSVs were marked positive when they were in the same direction as the outer inducing frame orientation and negative when they were in the opposite direction. Since no effect of frame direction (CW and $\mathrm{CCW}$ ) was detected in the experiment or in the following ones, the data from these conditions were pooled.

A repeated measures ANOVA was carried out with inducing condition (RFI or DRFI [RR]) and outer frame orientation $\left(11^{\circ}, 22^{\circ}\right.$, and $\left.33^{\circ}\right)$ as factors. Both the main effects and the interaction were significant $[F(1,20)=$ $8.465, p<.005 ; F(2,40)=7.39, p<.005 ; F(2,40)=6.03$, $p=.005$, respectively]. The subjects showed positive errors for small outer frame orientation $\left(11^{\circ}\right.$, mean error $=$ $0.5^{\circ}$ ), no effect at $22^{\circ}$ (mean error $\left.=-0.06^{\circ}\right)$, and negative (attraction) errors for larger outer frame orientation ( $33^{\circ}$, mean error $=-0.41^{\circ}$, in the RFI condition; only negative errors were found in the DRFI (RR) condition (mean errors $=-0.44^{\circ},-0.72^{\circ}$, and $-0.44^{\circ}$ at $11^{\circ}, 22^{\circ}$, and $33^{\circ}$ outer frame orientations, respectively). Positive signs for the illusion indicate repulsion effects, and negative signs indicate attraction effects. Post hoc comparisons showed a significant difference between inducing orientations in the RFI condition $(p<.005)$, but not in the DRFI (RR) condition. In particular, $11^{\circ}$ significantly differed from $22^{\circ}$ and $33^{\circ}$ outer frame orientation $(p<.005)$, but $22^{\circ}$ and $33^{\circ}$ did not reach significance $(p=.07)$. The RFI and DRFI (RR) means were different at $11^{\circ}$ and $22^{\circ}(p<$ $.005)$, but not at $33^{\circ}$.

\section{Discussion}

Our findings replicated the standard RFI angular function often reported before, showing repulsion and attraction effects for $11^{\circ}$ and $33^{\circ}$ tilts of the square frame, respectively; nevertheless, no systematic effect of the outer 
square frame orientation was evident in the DRFI (RR) condition. Although we replicated the attraction effect at $11^{\circ}$ outer frame orientation for the DRFI obtained by Zoccolotti et al. (1997), our results were not consistent with the predictions based on the hierarchical organization hypothesis. The DRFI (RR) effect did not vary with increasing outer frame orientation, and the error direction was always negative, opposite to the direction of the outer frame tilt. Moreover, the amplitude for the DRFI was not consistently smaller than that for the standard RFI, as had been suggested by Spinelli et al. (1999).

Are the results of Experiment 1 necessarily in conflict with an explanation in terms of global mechanisms and hierarchical organization theory? The hierarchical organization hypothesis implies that the outer frame influences the perception of the inner frame and only the latter frame induces an effect on the rod. This could still be the case, if we were to assume that the unexpected angular function in the DRFI condition (RR) was due to the effect of the outermost frame on the innermost frame.

In other words, the tilted outer frame could act differentially on the rod (in the RFI condition) and on the inner frame (in the DRFI [RR] condition), which, in turn, would affect the rod. This could be due to the differences in configuration (e.g., one-dimensional versus two-dimensional) between a test rod and a test square frame, so that the effect of an outer square frame on a test square frame could be determined not by the orientation of the outer frame's axes of symmetry, but by its real main-line axes defined by the sides of the frame (Wenderoth \& Beh, 1977). Contrary to this suggestion, Spinelli et al. (1999) tested this condition, using an $11^{\circ}$ outer frame tilt, and found no difference between a test rod and a test square in an otherwise standard RFI condition.

An alternative explanation could be given in terms of attended and unattended stimuli. Because the rod is the judged stimulus in both the RFI and the DRFI (RR) conditions, the computation of its orientation is different from that of the inner square orientation, which is not judged (and therefore, not attended) in the DRFI (RR) condition. We call the first a configurational hypothesis and the second an attentional hypothesis, and we tested them in the following experiments in order to give an account of the DRFI (RR) results.

\section{EXPERIMENT 2}

The aim of Experiment 2 was to measure the perceived orientation of the inner frame as a function of the outer frame orientation in a DRFI (RR) display.

The two conditions previously tested in Experiment 1, the standard RFI and the DRFI (RR), were tested again, and a new double-frame condition was used, in which the stimulus to be judged and the stimulus being varied were not the same. In particular, we tested a condition in which the inner frame was varied around the vertical axis and subjects were required to judge the rod, which was always objectively upright. This condition provided a measure of the effect of the inner frame in the absence of attention to it, a paradigm we will refer to as DRFI (FR). According to the definition of Reingold and Merikle (1988), "discriminations among a set of alternative stimulus states should be considered a direct measure of perception if the discriminative response is part of the task definition. ... Conversely, if the discriminative response is not part of the task definition, it should be considered an indirect measure of perception" (p. 564).

Thus, we measured both the tilt of the inner frame that results in the truly vertical rod being judged upright (DRFI [FR]) and the tilt of the rod that results in the rod itself being judged upright when the inner frame is truly upright (DRFI [RR]).

According to the hierarchical hypothesis, the inner frame should be influenced only by the outer frame, and the rod should, therefore, be perceived as vertical when it is tilted in the opposite direction from the outer frame for a $+11^{\circ}$ outer frame tilt but in the same direction as the outer frame for $\mathrm{a}+33^{\circ}$ outer frame tilt.

\section{Method}

Subjects. Twenty new students ( 12 of them male and 8 female) from the University of Rome "La Sapienza" participated in the experiment as volunteers. All had normal or corrected-to-normal vision.

Stimuli,Apparatus, and Procedure. The apparatus and stimuli were the same as those in Experiment 1.

Three experimental conditions were tested: two conditions, which were the same as those in Experiment 1, in which the rod, varying in orientation between $6^{\circ} \mathrm{CCW}$ and $6^{\circ} \mathrm{CW}$ in $1^{\circ}$ steps, was surrounded by one (standard RFI) or two (DRFI [RR]) square frames, and a third double-frame condition, in which the rod was always upright and the inner square frame orientation was varied between $6^{\circ} \mathrm{CCW}$ and $6^{\circ}$ $\mathrm{CW}$, in $1^{\circ}$ steps. We will refer to the new condition as DRFI (FR), where $\mathrm{F}$ indicates frame tilt variation.

The combination of three experimental conditions, 13 rod or inner frame tilts, and 6 outer frame orientations $\left(11^{\circ}, 22^{\circ}\right.$, and $33^{\circ}, \mathrm{CCW}$ or $\mathrm{CW}$ ) produced a total of 234 trials. The sequence of presentation was not blocked by condition.

The task was to judge rod orientation with a $2 \mathrm{AFC}$ method (left or right) in all three conditions. All the procedures were the same as those in Experiment 1.

\section{Results}

The data were analyzed as in Experiment 1, using the unidimensional Guttman scale for establishing the PSV. In the three conditions, the coefficients of reproducibility were all above 0.90 , indicating that the observed values were a good approximation of a perfect scale.

A repeated measures ANOVA was carried out with condition (RFI, DRFI [RR], or DRFI [FR]) and outer frame orientation $\left(11^{\circ}, 22^{\circ}\right.$, or $\left.33^{\circ}\right)$ as factors. The main effect of condition and the interaction were significant $[F(2,19)=$ $45.1, p<.0001$, and $F(4,38)=3.31, p<.0005$, respectively]. Whereas RFI and DRFI (RR) conditions replicated the results of Experiment 1, with the former having all positive errors and the latter all negative errors, the DRFI (FR) condition exhibited all positive errors (see Figure 3). Post hoc comparisons showed a significant difference between all the outer frame orientations in the RFI condition $(p<.05)$; no significant differences were shown in the DRFI (RR) condition, and only a difference between $22^{\circ}$ and $33^{\circ}$ was observed in the DRFI (FR) condition $(p<.05)$; the three conditions were different at $11^{\circ}$ 


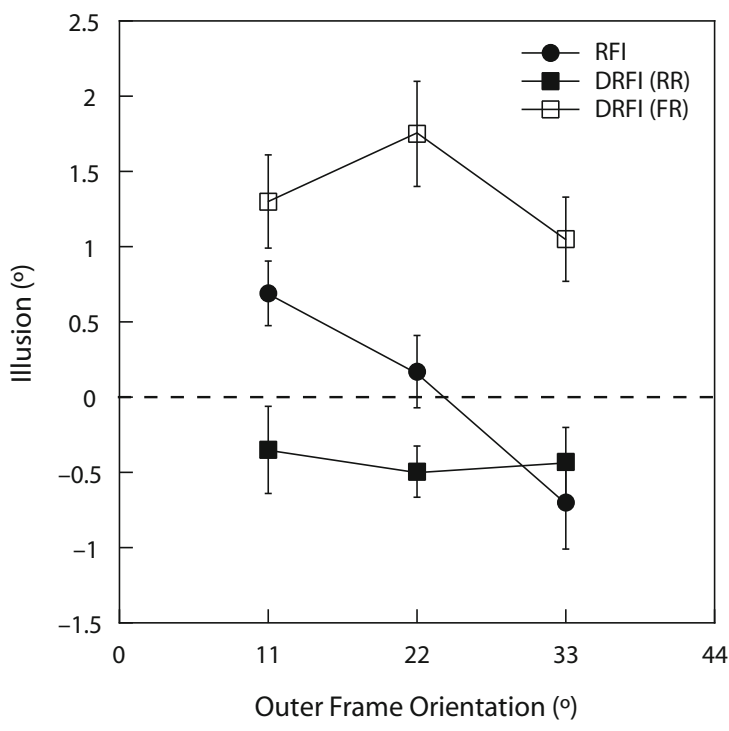

Figure 3. Means and standard errors of the illusions obtained in Experiment 2, as a function of outer frame orientation. A positive sign for the illusion stands for the repulsion effect, and a negative sign indicates an attraction effect.

and $22^{\circ}(p<.01)$, whereas only the DRFI (FR) condition differed significantly from the other two conditions at $33^{\circ}$.

It is interesting (see Figure 3) that in the DRFI (FR) condition, the PSV of the inner frame was always in the same direction as the outer frame orientation and in the direction opposite to the effect obtained in the DRFI (RR) condition. Moreover, the amplitude in the DRFI (FR) condition was larger $\left(\right.$ mean error $=1.37^{\circ}$ ) than those in the other two conditions $\left(0.05^{\circ}\right.$ and $-0.4^{\circ}$ were the mean errors in the RFI and DRFI [RR] conditions, respectively).

Although the subjects were not instructed to respond as quickly as possible, observation during data collection suggested a difference in response times between conditions. A second repeated measures ANOVA was carried out with response time (medians in milliseconds) as a dependent variable and condition (RFI, DRFI [RR], or DRFI [FR]) and outer frame orientation $\left(11^{\circ}, 22^{\circ}\right.$, or $\left.33^{\circ}\right)$ as factors. Outlying response times below $100 \mathrm{msec}$ and above $5,000 \mathrm{msec}$ were removed. Only the main effect of condition was significant $[F(2,40)=7.79, p=.001]$, and the response times and standard errors are shown in Figure 4.

The mean response time in the DRFI (FR) condition $(589.41 \mathrm{msec})$ was larger than the means in the other two conditions (RFI, $453.02 \mathrm{msec}$; DRFI [RR], $459.42 \mathrm{msec}$ ). There was no effect of outer frame orientation. Post hoc comparisons showed that the DRFI (FR) condition was different from the other two conditions $(p<.005)$.

\section{Discussion}

The results replicate all previous findings, showing that amplitude and direction of the effect is a function of outer frame orientation in the RFI condition, but not in the two DRFI conditions.
Moreover, the two DRFI conditions differed from each other: When rod orientation was varied and judged, the effect was in the direction opposite to the outermost frame orientation (attraction effect), whereas the opposite trend was observed when the inner frame orientation was varied and the rod was judged. Thus, the DRFI (FR) condition provides evidence that the outer frame induces on the inner frame an effect that is larger than that on the rod in the standard RFI and that is independent of the degree of tilt of the outer frame.

An inspection of the two functions of the double-frame conditions (Figure 3 ) suggested that the DRFI (RR) effects were highly related to the DRFI (FR) effects, so that the obtained DRFI (FR) illusions at each orientation could, in turn, account for each of the DRFI (RR) illusions. We suggest that the effect on the rod is completely due to the inner frame, as the hierarchical organization hypothesis would predict; nevertheless, we also propose that the effect of the inner square frame on the attended rod is different from the effect of an unattended inner square frame. We interpret these results as due to the different focus of attention, and the response time data are consistent with this view, showing a longer response time when the inner frame orientation is changed but attention is on the rod.

Another explanation for the difference observed in response times between the DRFI (FR) condition and the other two conditions is in terms of difficulty: In this condition, the subjects were required to judge the direction of the orientation of a rod that was physically vertical, whereas in the other conditions, there were difficult trials (those in which the rod was vertical) and easy trials (those in which the rod was tilted $4^{\circ}, 5^{\circ}$, or $6^{\circ}$ from the objective vertical).

Nevertheless, this second explanation would suggest that the subjects could discriminate when the rod was vertical. If this was the case, why did they exhibit a bigger

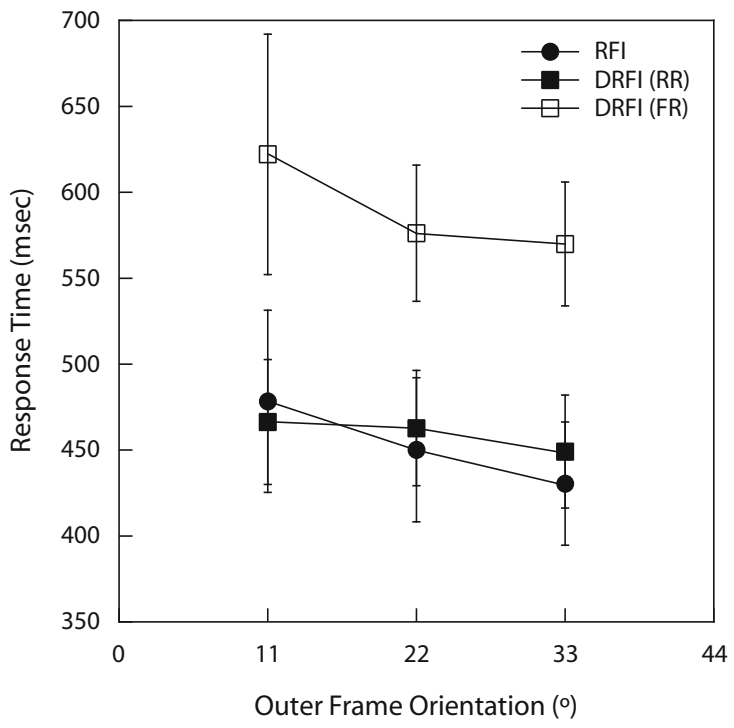

Figure 4. Medians of the response times in Experiment 2, as a function of outer frame orientation. 
illusory effect in the DRFI (FR) condition? Following this interpretation, indeed, we would have expected a random choice and no illusory effect.

\section{EXPERIMENT 3}

In Experiment 2, we indirectly tested the perceived orientation of the inner frame induced by a tilted external frame, and we found that the error was much bigger than that induced by the outermost frame on the rod. To claim that this was due to the focus of top-down attention, instead of the difference in shape between a square and a rod, we needed to compare the identical shape (a square) and configuration (DRFI) while varying the attended object. In the third experiment, we compared the effect of the outer frame on the perceived orientation of the inner frame in a direct and an indirect task. Our aim was to test whether the previous results were due to a difference in attending or not attending to the judged stimulus or to the effect of its shape (attentional vs. configurational hypothesis).

In this experiment, we manipulated top-down attention by comparing the same visual context and varying the task. The subjects had to judge the rod (DRFI [FR]) or the inner frame (DRFI $[\mathrm{FF}]$ ) with the same stimulus configuration. In this experiment, the subjects were instructed to respond both as quickly and as accurately as possible.

\section{Method}

Subjects. Twenty-two new students (11 of them male and 11 female) were drawn from the same population as those in Experiments 1 and 2 .

Stimuli, Apparatus, and Procedure. The stimuli and apparatus were the same as those used in Experiment 1: an upright rod surrounded by a single or a double frame, where the outermost frame could be tilted at $11^{\circ}, 22^{\circ}$, and $33^{\circ}, \mathrm{CCW}$ and $\mathrm{CW}$, while the inner frame for each outer frame orientation varied between $6^{\circ} \mathrm{CCW}$ and $6^{\circ} \mathrm{CW}$ in $1^{\circ}$ steps.

The subjects were required to judge the orientation of the rod in one presentation (RFI and DRFI [FR]) and the orientation of the inner frame in another presentation of the same DRFI (FF) stimuli.

The combination of three experimental conditions, 13 rod tilts, and six outer frame orientations produced a total of 234 trials. Half of the subjects started with one task (AB sequence), and half with the other (BA sequence). For each condition, the stimuli were presented to all the subjects in the same quasirandom sequence. The sequence of presentation was not blocked by condition.

Finally, the subjects were required to respond as accurately and quickly as possible.

\section{Results}

Mean illusions and standard errors are shown in Figure 5, and response times and their standard errors are shown in Figure 6.

As in Experiment 1, the data were analyzed by using the unidimensional Guttman scale for establishing the PSV. In the three conditions, the coefficients of reproducibility were all above 0.90 , indicating that the observed values are a good approximation of a perfect scale. Since no effect of the sequence ( $\mathrm{AB}$ and $\mathrm{BA})$ was detected in the experiment, the data from these conditions were pooled together.

A repeated measures ANOVA was carried out with condition (RFI, DRFI [FR], or DRFI [FF]) and frame orientation $\left(11^{\circ}, 22^{\circ}\right.$, or $\left.33^{\circ}\right)$ as factors. Both main effects were

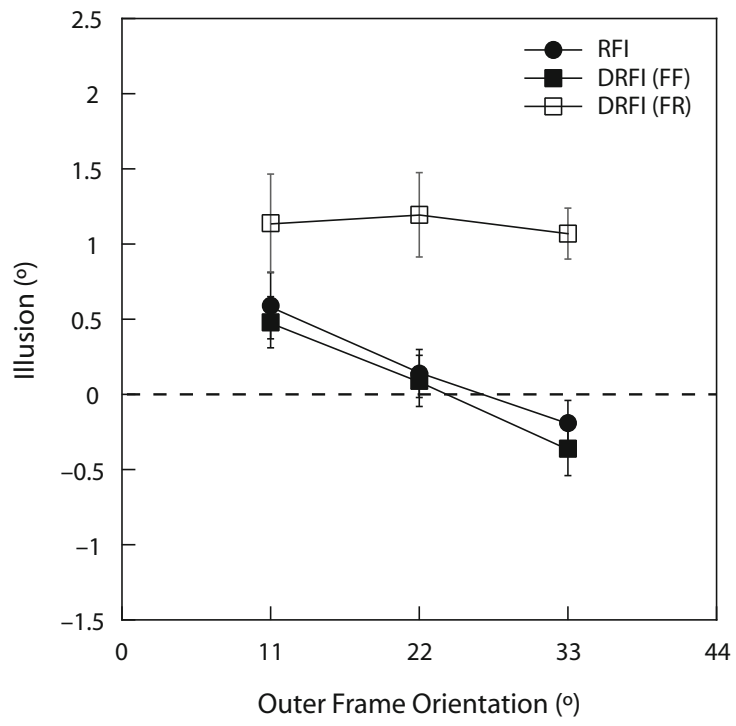

Figure 5. Means and standard errors of the illusions obtained in Experiment 3, as a function of outer frame orientation. A positive sign for the illusion stands for the repulsion effect, and a negative sign indicates an attraction effect.

significant $[F(1,21)=27.69, p<.0001$, and $F(2,42)=$ $4.27, p<.05$, for the condition and orientation factors, respectively]. The DRFI (FR) conditions induced a mean error $\left(1.13^{\circ}\right)$ much bigger than those in the other two conditions (both $0.18^{\circ}$ ). The smallest frame orientation induced the largest illusory effect $\left(0.74^{\circ}, 0.48^{\circ}\right.$, and $0.28^{\circ}$ for $11^{\circ}, 22^{\circ}$, and $33^{\circ}$ outer frame orientation, respectively).

Post hoc comparisons of the condition factor confirmed that DRFI (FR) significantly differed from RFI $(p<$

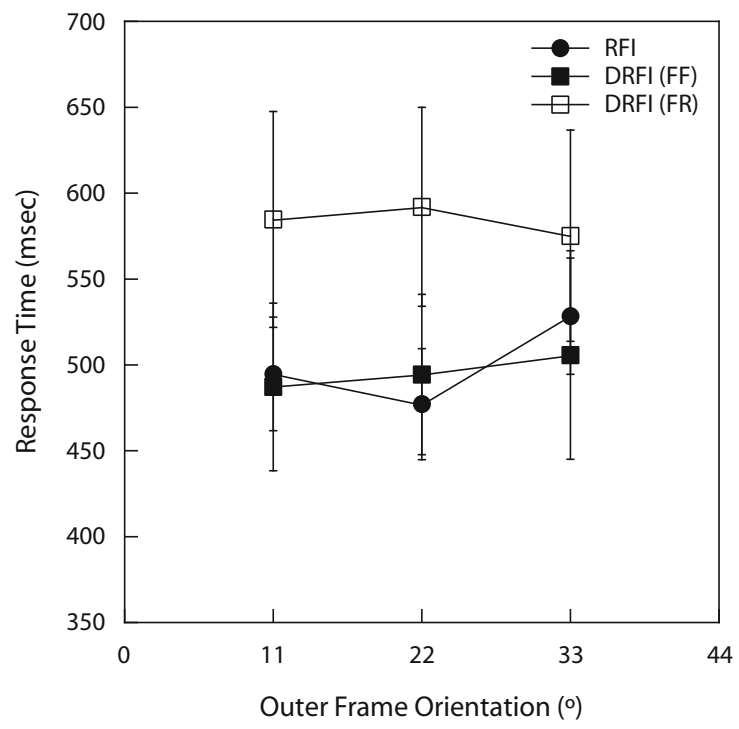

Figure 6. Medians of the response times in Experiment 3, as a function of outer frame orientation. 
$.0001)$ and DRFI (FF) $(p<.0001)$ but that the last two conditions did not differ from each other.

Post hoc comparisons of the orientation factor showed a difference between $11^{\circ}$ and $33^{\circ}(p<.01)$, whereas $22^{\circ}$ did not differ from the other two orientations.

A second repeated measures ANOVA was carried out with response time (in milliseconds) as a dependent variable and task (judging the rod or the inner frame) and frame orientation $\left(11^{\circ}, 22^{\circ}\right.$, or $\left.33^{\circ}\right)$ as factors. No significant effects were found, but an inspection of Figure 4 suggests that the large variance conceals a difference between the DRFI (FR) condition (longer response times) and the other two conditions (shorter response times): Planned comparisons showed a difference at $22^{\circ}[F(1,21)=179.29, p<.0001]$ and $33^{\circ}$ $[F(1,21)=165.28, p<.0001]$, but not at $11^{\circ}$.

\section{Discussion}

According to the alternative hypothesis that the effect induced by an external frame on a square is different from that induced on a rod and that this could explain the results of Experiment 2, we expected to obtain the same results from the DRFI (RR) and DRFI (FR) conditions, given that the inner frame orientation was manipulated in both conditions. On the contrary, using the same stimuli and varying the task, the results were different in amplitude, direction, and response time. No differences were found between the RFI and the DRFI (FF) conditions, which suggests that the effect of a tilted outer frame on a square is around the same as that on a rod.

The results of the third experiment, therefore, confirmed our hypothesis that the results of the previous experiments were due to different processing of the same stimulus condition in the indirect and the direct measures of the inner frame orientation.

We are suggesting that the hierarchical organization effect in the DRFI (RR) condition is due to different mechanisms arising from the same visual context, whose influence depends on attentional modulation.

\section{EXPERIMENT 4}

The data of Experiments 1, 2, and 3 were collected with a somewhat imprecise psychophysical scaling method (e.g., Spinelli, Antonucci, Goodenough, Pizzamiglio, \& Zoccolotti, 1991), and even if we replicated all the results, we wanted to be sure that they were not due merely to the imprecision of the technique. In this experiment, therefore, we repeated the two conditions that gave unexpected results (DRFI [RR] and DRFI [FR]) with a different, more accurate psychophysical technique.

\section{Method}

Subjects. Eighteen new students ( 9 of them male and 9 female) from Macquarie University, Sydney, participated in the experiment as volunteers. All had normal or corrected-to-normal vision.

Stimuli, Apparatus, and Procedure. Stimulus displays were presented on a Silicon Graphics D-M2ONC 19-in. color display monitor (frame rate $=120 \mathrm{~Hz}$, interlaced frame rate $=60 \mathrm{~Hz}$ ) interfaced with a Silicon Graphics Indigo 2 workstation. Special-purpose software was designed and written to control stimulus presentation conditions during the experiment.
During the test conditions, a central rod $1^{\circ}$ long and $0.1^{\circ}$ thick was surrounded by two larger square frames of the same thickness as the test rod. The sides of the inner frame were $3^{\circ}$ long, and the sides of the outer frame were $7^{\circ}$ long. The gaps between the rod and the inner frame and between the inner and the outer frames were never smaller than $1^{\circ}$, to eliminate lateral inhibitory interactions (see Wenderoth \& Johnstone, 1988). All the stimuli were constructed with Adobe Photoshop.

The stimuli were presented for $500 \mathrm{msec}$, with an interstimulus interval of $3 \mathrm{sec}$.

The subjects' responses were recorded using the outer pair of buttons on a three-button mouse to indicate whether the stimulus target appeared to be tilted to the left or the right of perceived vertical. The subjects were seated in a darkened laboratory in which all external cues to vertical were removed by attaching to the display monitor a black cardboard $9^{\circ}$-diameter mask. A black cloth was draped over the area between the display and a padded chinrest on which the subjects placed their heads. The chinrest was located $1.14 \mathrm{~m}$ from the display, so that $2 \mathrm{~cm}$ on the screen subtended $1^{\circ}$ of visual angle.

Each subject was tested under three conditions with an outer, inducing square frame: $11^{\circ}, 22^{\circ}$, or $33^{\circ} \mathrm{CW}$.

Prior to each set of trials on which both test and inducing fields were presented simultaneously (called the test condition), the subjects were run under a pretest condition in which the test field alone was presented and orientation judgments of it were made. A pretest condition always preceded a test condition to control for possible drift in subjective vertical over the experiment. A double, randomly interleaved staircase technique was used, with two staircase measures taken under each condition. Each staircase was started from a test stimulus orientation randomly chosen from the range $\pm 10^{\circ}$ from gravitational vertical. Step size was initially $2^{\circ}$ and was reduced to $1^{\circ}$ after the third reversal in decision. Staircases were run for 10 reversals, with the PSV estimated by averaging the peaks and valleys of the last 6 reversals. The subjects were instructed to be as accurate as possible in their judgments and to maintain fixation on a small dark spot in the center of the display during each trial. In the first condition (DRFI [RR]), the inner frame was always vertical, the variable stimulus was the rod, and the subjects were required to judge the rod, whereas in the second condition (DRFI [FR]), the variable stimulus was the inner frame, but the subjects were asked to judge the rod (always vertical). Following the 500-msec presentation duration of each trial, the entire screen was blanked to $6.4 \mathrm{~cd} / \mathrm{m}^{2}$, which was sufficient to eradicate any afterimages. Only in this experiment was the sequence of presentation blocked by condition. The entire session lasted approximately $30 \mathrm{~min}$.

\section{Results}

The dependent variable was the PSV in degrees, estimated by subtracting threshold values for the pretest from those in the experimental conditions.

A repeated measures ANOVA was carried out with condition (DRFI [FR] or DRFI [RR]) and frame orientation $\left(11^{\circ}, 22^{\circ}\right.$, or $\left.33^{\circ}\right)$ as factors. The main effect of condition attained significance $[F(1,17)=30.22, p<.0001]$, whereas the main effect of orientation and the interaction effect did not.

As in Experiment 2 (see Figure 7), the effect in the DRFI (FR) condition was always in the same direction as the outer frame orientation and in the direction opposite to the effect obtained in the DRFI (RR) condition; finally, the former was larger in amplitude than was the latter $\left(1.79^{\circ}\right.$ vs. $-0.68^{\circ}$, respectively $)$.

\section{Discussion}

Experiment 4 confirmed the results of Experiment 2, showing a small $\mathrm{CCW}$ effect when the rod was attended 


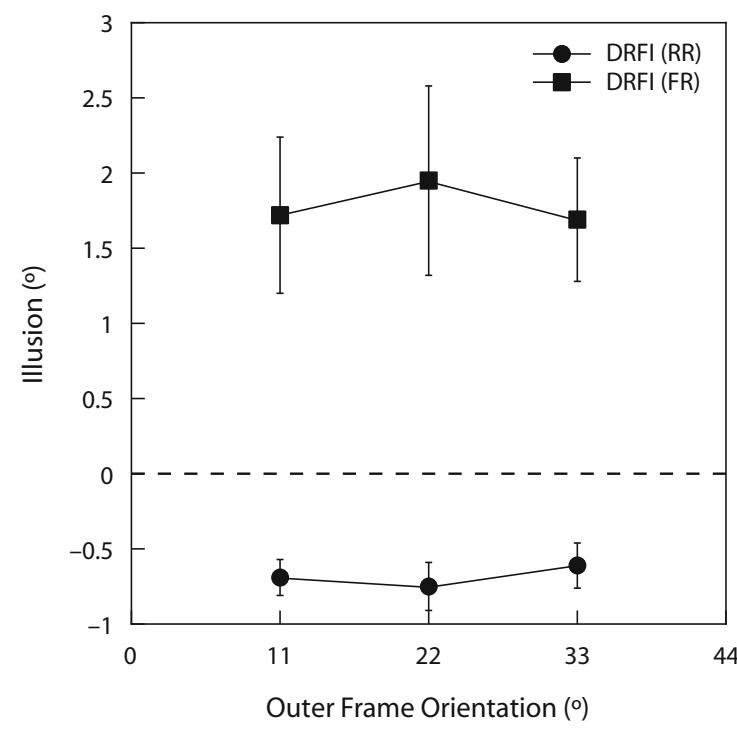

Figure 7. Means and standard errors of the illusions obtained in Experiment 4, as a function of outer frame orientation. A positive sign for the illusion stands for the repulsion effect, and a negative sign indicates an attraction effect.

and its perceived orientation was measured and a large $\mathrm{CW}$ effect when the rod was attended but the perceived orientation of the inner frame was measured. In neither case, again, did the illusory effect vary as a function of the outermost frame orientation. The similarity of the results between Experiments 2 and 4 confirmed the validity and the reliability of our results.

\section{GENERAL DISCUSSION}

The data exhibited an unexpected angular function when an inner vertical frame was added to the standard rod-andframe display. In fact, in the DRFI (RR), the outer frame induces an effect on the rod in such a way that the error is consistently in the direction opposite the frame's orientation, but this is not a direct effect, which would predict a standard RFI function. It is also not a simple hierarchical effect from the outermost to the innermost stimulus, which would predict an angular function opposite to that of the standard RFI, and smaller errors as well.

We tested alternative hypotheses: first, a configurational hypothesis that the outer frame induces different effects on the inner frame and the rod because they differ in shape and, second, an attentional hypothesis that the outer frame acts differently on attended and unattended stimuli.

Both the configurational and the attentional hypotheses seem useful in accounting for the DRFI (RR) angular function. The configurational hypothesis explains why the effect does not vary across orientations of the outer frame, because only the real main-line axes are relevant to the task when both inducing and test stimuli are twodimensional (Daini et al., 2003). The second explains why the effect is different when more than one frame induces an effect on the rod and why the size of the outer frame effect on the internal frame is bigger than what would be expected from the standard RFI effect.

We took direct (Experiment 3) and indirect (Experiment 2,3, and 4) measures of the perceived orientation of the objectively upright inner frame. We found that whereas the direct measure of the inner frame orientation is similar to that for the standard RFI (Experiment 3), the indirect measure of it is completely different (Experiments 2 and 4). The former result is contrary to the configurational hypothesis, whereas the latter is congruent with the attentional hypothesis, suggesting that the perceived orientation on the unattended inner frame can explain the perceived orientation of the attended rod in the DRFI.

In our results, the effect of top-down attention is clear: When the subjects judged exactly the same stimulus configuration, but with different tasks - judging the frame versus judging the rod-illusions differed in both size and direction. Moreover, the very much longer response times in the DRFI (FR) condition, as compared with the other conditions in Experiments 2 and 3, strongly suggest that different mechanisms were in operation.

The hypothesis that the top-down attention has a modulatory effect on (bottom-up) illusory phenomena has been suggested in different contexts.

In neuropsychology, evidence has come from patients with unilateral spatial neglect (USN), who, although they are not able to pay attention to those stimuli presented in the contralesional side of space, show preserved illusory perception for both sides of space (e.g., Mattingley, Bradshaw, \& Bradshaw, 1995; Olk \& Harvey, 2002; Ro \& Rafal, 1996; Vallar, Daini, \& Antonucci, 2000). Furthermore, the size of the illusion is even greater for the contralesional "neglected" hemispace (Chatterjee \& Thompson, 1998; Daini, Angelelli, Antonucci, Cappa, \& Vallar, 2002; Vallar \& Daini, 2006).

Our speculation about the mechanisms underlying the unexpected results obtained with the DRFI (RR) are as follows. Bottom-up processes induce visual illusions of orientation, as has been demonstrated in other illusory contexts (see Vallar \& Daini, 2006). The illusion is due to the global mechanism described by Beh et al. (1971): The outer frame orientation, through the extraction of its mainline axes of symmetry, is used as a reference for computing the orientation of the test stimulus, and these are repulsion effects from the real main lines of the frame. This mechanism gives the relative orientation of objects (in a way that is similar to those of the mechanisms underlying color or size perception) and acts hierarchically from the outermost to the innermost stimulus in a serial, additive way. However, when the task invokes top-down processing, so that subjects are instructed to compute the orientation of the test stimulus relative to gravitational vertical, this affects the illusion in two ways: When the inducer orientation is between $22.5^{\circ}$ and $45^{\circ}$, the virtual diagonal symmetry axes are closer to vertical than are the main-line axes and, hence, are more relevant to the task than are the main-line axes. Moreover, attentional top-down modulation reduces the amount of illusory effects, as has been shown in neglect patients who exhibit larger illusions for 
the neglected side of space where attention is not operating (Daini et al., 2002).

In other words, in the standard rod-and-frame test, with central displays and a large gap between the rod and the frame, bottom-up processing determines that the orientation of the rod is computed in reference to the main-line axes of the frame. On the other hand, top-down processing, required by the instruction to judge in terms of gravitational vertical, results in the effect of whichever axis, main-line or virtual diagonal, is closest to true vertical.

When more than one frame affects the rod stimulus, there are also effects between the frames themselves, which are not related to the task and, consequently, to topdown attention. The effect of the outer frame on the inner frame is not computed relative to the vertical axis when the inner frame itself is not the stimulus being judged.

Finally, in both the RFI and the DRFI (RR), the influence is always from the outermost to the innermost stimulus, and each stimulus is affected only by the closest, as the hierarchical organization theory predicts.

These results are interesting from three points of view. First, in the study of the illusory effects of orientation and, more generally, in the visual perception of verticality, they contribute to the explanation of the hierarchical organization phenomenon, showing the influence of attention on the global mechanisms of shape and orientation analysis. Second, in the study of attention, the results offer a new paradigm: The double frame looks like a new and interesting experimental paradigm with which to study the relation between the processing of spatial coordinates and attention, because it produces directional differences, rather than mere quantitative differences, in the measurement of these.

Finally, there is recent evidence that the magnitude of illusions, and even whether they occur at all, depends on which of two tasks is given to the observer, even when the visual display is identical in the two different task conditions (Jazayeri \& Movshon, 2007). This is essentially what we have found, and the recent emphasis on the Bayesian approach to sensory decoding (Jazayeri \& Movshon, 2006; Weiss, Simoncelli, \& Adelson, 2002) may well be relevant to the effects that we have reported.

\section{AUTHOR NOTE}

Correspondence concerning this article should be addressed to R. Daini, Dipartimento di Psicologia, Università degli Studi di MilanoBicocca, via dell'Innovazione 10, 20126 Milan, Italy (e-mail: roberta .daini@unimib.it).

\section{REFERENCES}

Beh, H. C., Wenderoth, P. M., \& Purcell, A. T. (1971). The angular function of a rod-and-frame illusion. Perception \& Psychophysics, 9, 353-355.

Chatterjee, J., \& Thompson, K. A. (1998). Weigh(t)ing for awareness. Brain \& Cognition, 37, 477-490.

Daini, R., Angelelli, P., Antonucci, G., Cappa, S. F., \& Vallar, G.
(2002). Exploring the syndrome of spatial unilateral neglect through an illusion of length. Experimental Brain Research, 144, 224-237.

Daini, R., Wenderoth, P. [M.], \& SMith, S. (2003). Visual orientation illusions: Global mechanisms involved in hierarchical effects and frames of reference. Perception \& Psychophysics, 65, 770-778.

Dunn-Rankin, P. (1983). Scaling methods. Hillsdale, NJ: Erlbaum.

JAZAYERI, M., \& MOVSHON, J. A. (2006). Optimal representation of sensory information by neural populations. Nature Neuroscience, 9, 690-696.

JAZAYERI, M., \& Movshon, J. A. (2007). A new perceptual illusion reveals mechanisms of sensory decoding. Nature, 446, 912-915.

Mattingley, J. B., Bradshaw, J. L., \& Bradshaw, J. A. (1995). The effects of unilateral visuospatial neglect on perception of Müller-Lyer illusory figures. Perception, 24, 415-433.

OlK, B., \& HarveY, M. (2002). Effects of visible and invisible cueing on line bisection and Landmark performance in hemispatial neglect. Neuropsychologia, 40, 282-290.

Reingold, E. M., \& MerikLe, P. M. (1988). Using direct and indirect measures to study perception without awareness. Perception \& Psychophysics, 44, 563-575.

Ro, T., \& Rafal, R. D. (1996). Perception of geometric illusions in hemispatial neglect. Neuropsychologia, 34, 973-978.

Rock, I. (1974). The perception of disoriented figures. Scientific American, 230, 78-85.

Rock, I. (1990). The frame of reference. In I. Rock (Ed.), The legacy of Solomon Asch: Essays in cognition and social psychology (pp. 243268). Hillsdale, NJ: Erlbaum.

Spinelli, D., Antonucci, G., Daini, R., Martelli, M. L., \& ZoccoLOTTI, P. (1999). Hierarchical organisation in perception of orientation. Perception, 28, 965-979.

Sinelli, D., Antonucci, G., Goodenough, D. R., Pizzamiglio, L., \& Zoccolotti, P. (1991). Psychophysiological mechanisms underlying the rod and frame illusion. In S. Wapner \& J. Demick (Eds.), Field dependence-independence: Cognitive style across the life span (pp. 37-60). Hillsdale, NJ: Erlbaum.

VALlaR, G., \& DainI, R. (2006). Visual perceptual processing in unilateral spatial neglect: The case of visual illusions. In T. Vecchi \& G. Bottini (Eds.), Imagery and spatial cognition: Methods, models, and cognitive assessment (pp. 337-362). Amsterdam: Benjamins.

VAllar, G., DainI, R., \& ANTONUCCI, G. (2000). Processing of illusion of length in spatial hemineglect: A study of the line bisection. Neuropsychologia, 38, 1087-1097.

Weiss, Y., Simoncelli, E. P., \& Adelson, E. H. (2002). Motion illusions as optimal percepts. Nature Neuroscience, 5, 598-604.

WENDEROTH, P. M. (1974). The distinction between the rod-and-frame illusion and the rod-and-frame test. Perception, 3, 205-212.

Wenderoth, P. [M.], \& Beh, H. (1977). Component analysis of orientation illusions. Perception, 6, 57-75.

Wenderoth, P. [M.], \& Johnstone, S. (1987). Possible neural substrates for orientation analysis and perception. Perception, 16, 693-709.

Wenderoth, P. [M.], \& Johnstone, S. (1988). The different mechanisms of the direct and indirect tilt illusions. Vision Research, 28, 301-312.

Wenderoth, P. [M.], van der Zwan, R., \& Johnstone, S. (1989). Orientation illusions induced by briefly flashed plaids. Perception, 18, 715-728.

Wenderoth, P. [M.], van der Zwan, R., \& Williams, M. (1993). Direct evidence for competition between local and global mechanisms of two-dimensional orientation illusions. Perception, 22, 273-286.

WitKIN, H. A., \& Asch, S. E. (1948). Studies in space orientation: IV. Further experiments on perception of the upright with displaced visual fields. Journal of Experimental Psychology, 38, 762-782.

Zoccolotti, P., Antonucci, G., Daini, R., Martelli, M. L., \& SPINELLI, D. (1997). Frame-of-reference and hierarchical-organisation effects in the rod-and-frame illusion. Perception, 26, 1485-1494.

(Manuscript received September 20, 2007; revision accepted for publication April 14, 2008.) 